\title{
Seroepidemiology of infection with Toxoplasma gondii in migrant agricultural workers living in poverty in Durango, Mexico
}

\author{
Cosme Alvarado-Esquivel ${ }^{1 *}$, Federico Campillo-Ruiz ${ }^{2}$ and Oliver Liesenfeld ${ }^{3,4}$
}

\begin{abstract}
Background: Migrant agricultural workers are a group of people living in poverty with poor housing, sanitary conditions and hygiene practices. Little is known about the epidemiology of infection with Toxoplasma gondii in migrant agricultural workers.

Methods: We investigated the presence of anti-Toxoplasma IgG and IgM antibodies in 173 migrant workers hired for seasonal agricultural work in Durango State in northern Mexico using enzyme-linked immunoassays.

Results: Of the 173 migrant workers (mean age 34.82 \pm 14.01 years), 50 (28.9\%) had anti-Toxoplasma lgG antibodies and 36 (20.8\%) had anti-Toxoplasma IgM antibodies. Seroprevalence was not influenced by gender, age, birth place, or educational level. In contrast, seroprevalence was significantly higher in workers residing in rural areas than those in urban or suburban areas. Migrant workers suffering from memory impairment, dizziness, or syncope had significantly higher seroprevalence of anti-T. gondii lgG antibodies than those without such clinical features. Logistic regression analysis showed that $T$. gondii exposure was positively associated with consumption of unwashed raw vegetables $(\mathrm{OR}=2.39 ; 95 \% \mathrm{Cl}: 1.06-5.35 ; P=0.03)$ and low frequency of eating out of home $(\mathrm{OR}=3.87 ; 95 \% \mathrm{Cl}: 1.43-10.42$; $P=0.007)$, and negatively associated with national trips (OR $=0.30 ; 95 \% \mathrm{Cl}: 0.13-0.65 ; P=0.003)$ and consumption of raw milk ( $\mathrm{OR}=0.40 ; 95 \% \mathrm{Cl}: 0.18-0.87 ; P=0.02)$. Other behavioral characteristics including consumption of meat or untreated water were not associated with $T$. gondii infection.

Conclusions: This is the first report of T. gondii infection in internal migrant agricultural workers living in poverty. Results deserve further investigation of causal relations between clinical symptoms and infection, and may be useful for optimal planning of preventive measures.
\end{abstract}

Keywords: Toxoplasma gondii, Seroprevalence, Migrant workers, Cross-sectional study

\section{Background}

Toxoplasma gondii (T. gondii) is a parasite with worldwide distribution [1]. Infections with T. gondii may lead to serious illness affecting mostly lymph nodes, eyes, and the central nervous system [2-4]. Transmission of T. gondii occurs by ingesting food or water contaminated with oocysts shed by cats or by eating undercooked or raw meat containing tissue cysts [2]. There is poor knowledge about the epidemiology of $T$. gondii infection in migrant agricultural workers in the world in general [5-7], and we are not

\footnotetext{
* Correspondence: alvaradocosme@yahoo.com

'Laboratorio de Investigación Biomédica, Facultad de Medicina y Nutrición, Universidad Juárez del Estado de Durango. Avenida Universidad S/N,

Durango, Dgo 34000, México

Full list of author information is available at the end of the article
}

aware of any report in the medical literature about the epidemiology of T. gondii infection in migrant workers in Mexico in particular. It is important to study migrant agricultural workers since they live in poverty, under poor sanitation conditions and low hygiene practices. They use untreated drinking water and have poor health care services for diagnosis, treatment, and prevention of infectious diseases in general and a lack of laboratory tests for toxoplasmosis in particular. Previous studies in Durango have shown higher frequencies of $T$. gondii exposure in rural populations $[8,9]$ than in inhabitants in the urban capital city [10-12]. Therefore, we sought to determine the seroprevalence of $T$. gondii exposure in migrant agricultural

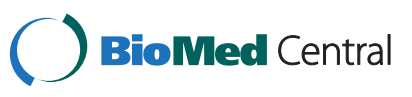


workers in Durango, Mexico and to identify their characteristics associated with Toxoplasma seropositivity.

\section{Methods}

\section{Study design and study population}

We performed a cross sectional survey from August 2010 to August 2012 in national (internal) migrant workers hired for seasonal agricultural work in Durango State, Mexico. Migrant agricultural workers in Mexico consist of a particular group of the population who move from region to region within the country to get agricultural work. Many of these itinerant agricultural workers travel from one region to another along with their family members. Local (born in the same Mexican state where they work) migrant agricultural workers outnumber those who migrate from other Mexican states or from abroad. In Durango, migrant agricultural workers come from several regions of Durango State and neighboring states from northern Mexico. The migration patterns of these workers were mostly rural-rural and few were urban-rural. During their temporary employment, migrant workers live in poor socioeconomic conditions including poor housing, food, and sanitation. Inclusion criteria for the study subjects were: 1) migrant workers involved in seasonal agricultural labor in Durango, Mexico, 2) any gender, 3) 14 years and older, 4) any socioeconomic level, and 5) that voluntarily accepted to participate. In total, 173 migrant agricultural workers were studied. They worked in the municipalities of Durango and Canatlán in the valley region of Durango State. Durango municipality has a temperate climate and Canatlán municipality has a semi-cold climate. Migrant agricultural workers had spent up to 3 months in the current work place.

\section{Ethical aspects}

This study was approved by the Institutional Ethical Committee of the General Hospital of the Secretary of Health in Durango City. The purpose and procedures of the study were explained to all participants. A written informed consent was obtained from all participants.

\section{Socio-demographic, clinical, and behavioral data}

We obtained the characteristics of the participants by using a standardized questionnaire. Socio-demographic data including age, gender, birth place, residence, educational level, and socioeconomic status were obtained from all participants. Clinical data explored included the presence of underlying diseases, presence or history of lymphadenopathy, frequent presence of headache, memory, reflex, hearing, and visual impairments, and a history of surgery, blood transfusion or transplants; in women, a history of abortion was also documented. Contributing and confounding risk factors of behavioral data included animal contacts, contact with cat excrement, foreign travel, type of meat consumption, frequency of meat consumption, consumption of raw or undercooked meat, unpasteurized milk, dried or cured meat, consumption of unwashed raw vegetables, fruits, or untreated water, frequency of eating out of home (in restaurants or fast food outlets), contact with soil, and types of floors at home were obtained from all participants.

\section{Serological examination for T. gondii antibodies}

Serum samples were obtained from all participants and kept frozen at $-20^{\circ} \mathrm{C}$ until analyzed. Serum samples were analyzed by qualitative and quantitative methods for anti- $T$. gondii IgG antibodies with a commercially available enzyme immunoassay "Toxoplasma IgG" kit (International Immuno-Diagnostics, Foster City, CA, U.S.A.). Anti-T. gondii IgG antibody levels were expressed as International Units (IU)/ml, and a result equal to or greater than $8 \mathrm{IU} / \mathrm{ml}$ was considered positive. In addition, sera positive for T. gondii IgG were further analyzed for anti-T. gondii IgM antibodies by a commercially available enzyme immunoassay "Toxoplasma IgM" kit (International ImmunoDiagnostics, Foster City, CA, U.S.A.). Such a strategy of testing anti-T. gondii IgM antibody only in anti-T. gondii IgG antibody positive individuals, and not vice versa, was selected because anti- $T$. gondii IgM antibodies disappear with time while anti- $T$. gondii IgG antibodies appear early after infection and remain over a lifetime. All tests were performed following the instructions of the manufacturer.

\section{Statistical analysis}

The statistical analysis was performed with the aid of the software Epi Info version 3.5.3 and SPSS version 15.0. For calculation of the sample size, we used a reference [8] seroprevalence of $23.8 \%$ as expected frequency of the factor under study, 1,000 as the size of the population from which the sample was selected, a least acceptable result of $30.0 \%$, and a confidence level of $95 \%$. The result of the calculation was 153 subjects. We used the Pearson's chi-square test and the Fisher exact test (when values were less than 5) for comparison of the frequencies among groups. Bivariate and multivariate analyses were used to assess the association between the characteristics of the subjects and T. gondii seropositivity. Variables were included in the multivariate analysis if they had a $P$ value equal to or less than 0.20 in the bivariate analysis. Odds ratio (OR) and $95 \%$ confidence intervals $(\mathrm{CI})$ were calculated by multivariate analysis using multiple, unconditional logistic regression. A $P$ value less than 0.05 was considered statistically significant.

\section{Results}

Of the 173 migrant workers, 50 (28.9\%) had anti-T. gondii IgG antibodies and 36 (20.8\%) were also positive for anti$T$. gondii IgM antibodies. Of the 50 anti-T. gondii IgG positive participants, 31 (62\%) had IgG levels higher than 
$150 \mathrm{IU} / \mathrm{ml}, 2$ (4\%) between 100 to $150 \mathrm{IU} / \mathrm{ml}$, and 17 (34\%) between 9 to $99 \mathrm{IU} / \mathrm{ml}$. General socio-demographic characteristics of the 173 migrant workers and their relation with $T$. gondii seropositivity are shown in Table 1 . All workers had a low socioeconomic status. Most participants were born in Durango, Mexico. The mean age of participants was $34.82 \pm 14.01$ years (range 14-89 years). Seroprevalence of $T$. gondii exposure was not significantly influenced by age, gender, birth place, or educational level. In contrast, the seroprevalence of $T$. gondii infection was significantly higher in migrant workers residing in rural areas than those in urban or suburban areas $(P=0.04)$.

With respect to clinical data (Table 2), migrant workers suffering from memory impairment had significantly higher seroprevalences of anti- $T$. gondii IgG than those without this clinical feature $(P=0.007)$. In addition, migrant workers suffering from dizziness or syncope had significantly higher seroprevalence of anti- $T$. gondii IgG antibodies than those without such clinical characteristics $(P=0.02)$. The frequencies of other clinical characteristics including presence or history of lymphadenopathy, reflex, hearing and visual impairments, surgery history, blood transfusion, and transplant history were similar among

Table 1 Socio-demographic characteristics of migrant agricultural workers and seroprevalence of $T$. gondii infection

\begin{tabular}{|c|c|c|c|c|}
\hline \multirow[t]{2}{*}{ Characteristic } & \multirow{2}{*}{$\frac{\text { No. of subjects }}{\text { tested }^{\mathrm{a}}}$} & \multicolumn{2}{|c|}{$\begin{array}{c}\text { Prevalence of } \\
\text { T. gondii infection }\end{array}$} & \multirow{2}{*}{$\begin{array}{c}P \\
\text { value }\end{array}$} \\
\hline & & No. & $\%$ & \\
\hline \multicolumn{5}{|l|}{ Gender } \\
\hline Male & 95 & 29 & 30.5 & 0.6 \\
\hline Female & 78 & 21 & 26.9 & \\
\hline \multicolumn{5}{|l|}{ Age groups (years) } \\
\hline 30 or less & 79 & 17 & 21.5 & 0.14 \\
\hline $31-50$ & 68 & 24 & 35.3 & \\
\hline$>50$ & 26 & 9 & 34.6 & \\
\hline \multicolumn{5}{|l|}{ Birth place } \\
\hline Durango State & 160 & 47 & 29.4 & 0.75 \\
\hline Other Mexican State & 13 & 3 & 23.1 & \\
\hline \multicolumn{5}{|l|}{ Residence place } \\
\hline Durango State & 169 & 50 & 29.6 & 0.32 \\
\hline Other Mexican State & 4 & 0 & 0.0 & \\
\hline \multicolumn{5}{|l|}{ Residence area } \\
\hline Urban or suburban & 25 & 3 & 12.0 & 0.04 \\
\hline Rural & 147 & 47 & 32.0 & \\
\hline \multicolumn{5}{|l|}{ Educational level } \\
\hline No education & 35 & 7 & 20.0 & 0.53 \\
\hline $1-6$ years & 81 & 25 & 30.9 & \\
\hline $7-12$ years & 56 & 18 & 32.1 & \\
\hline 13 or more years & 1 & 0 & 0.0 & \\
\hline
\end{tabular}

${ }^{\mathrm{a}}$ Sums may not add up to 173 because of a missing value.
T. gondii positive and T. gondii negative individuals. Abortion history in women was not associated with $T$. gondii seropositivity. With respect to IgM seroprevalence, further analysis of clinical data showed that workers suffering from memory impairment also had a significantly $(P=0.02)$ higher seroprevalence of anti- $T$. gondii IgM antibodies (16/51: 31.4\%) than those without such clinical features

Table 2 Bivariate analysis of clinical data and infection with $T$. gondii in migrant agricultural workers of Durango State, Mexico

\begin{tabular}{|c|c|c|c|c|}
\hline \multirow{2}{*}{ Characteristic } & \multirow{2}{*}{$\frac{\text { No. of subjects }}{\text { tested }^{\mathrm{a}}}$} & \multicolumn{2}{|c|}{$\begin{array}{c}\text { Prevalence of } \\
\text { T. gondii infection }\end{array}$} & \multirow{2}{*}{$\begin{array}{c}P \\
\text { value }\end{array}$} \\
\hline & & No. & $\%$ & \\
\hline \multicolumn{5}{|l|}{ Clinical status } \\
\hline Healthy & 140 & 39 & 27.9 & 0.53 \\
\hline\|\| & 33 & 11 & 33.3 & \\
\hline
\end{tabular}

Lymphadenopathy ever

\begin{tabular}{ccccc}
\hline Yes & 45 & 14 & 31.1 & 0.70 \\
\hline No & 128 & 36 & 28.2 & \\
\hline Headache frequently & & & & \\
\hline Yes & 74 & 27 & 36.5 & 0.05 \\
\hline No & 99 & 23 & 23.2 & \\
\hline
\end{tabular}

Dizziness or syncope

\begin{tabular}{ccccc}
\hline Yes & 5 & 4 & 80 & 0.02 \\
\hline No & 168 & 46 & 27.4 & \\
\hline
\end{tabular}

\begin{tabular}{ccccc}
\hline Memory impairment & & & & \\
\hline Yes & 51 & 22 & 43.1 & 0.007 \\
\hline No & 122 & 28 & 23 & \\
\hline Reflex impairment & & & & \\
\hline Yes & 36 & 13 & 36.1 & 0.28 \\
\hline No & 137 & 37 & 27 & \\
\hline
\end{tabular}

Hearing impairment

\begin{tabular}{ccccc}
\hline Yes & 19 & 5 & 26.3 & 0.79 \\
\hline No & 154 & 45 & 29.2 & \\
\hline Visual impairment & & & & \\
\hline Yes & 48 & 18 & 37.5 & 0.12 \\
\hline No & 125 & 32 & 25.6 & \\
\hline Surgery ever & & & & \\
\hline Yes & 40 & 10 & 25 & 0.51 \\
\hline No & 132 & 40 & 30.3 & \\
\hline
\end{tabular}

Blood transfusion

\begin{tabular}{ccccc}
\hline Yes & 23 & 8 & 34.8 & 0.50 \\
\hline No & 150 & 42 & 28 & \\
\hline Transplantation & & & & \\
\hline Yes & 2 & 1 & 50 & 0.49 \\
\hline No & 171 & 49 & 28.7 & \\
\hline Abortion in women & & & & \\
\hline Yes & 19 & 5 & 26.3 & 0.58 \\
\hline No & 55 & 14 & 25.5 & \\
\hline
\end{tabular}

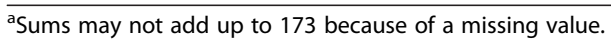


Table 3 Bivariate analysis of selected putative risk factors for infection with $T$. gondii in migrant agricultural workers in Durango, Mexico

\begin{tabular}{|c|c|c|c|c|}
\hline \multirow[t]{2}{*}{ Characteristic } & \multirow{2}{*}{$\begin{array}{c}\begin{array}{c}\text { No. of } \\
\text { subjects }\end{array} \\
\text { tested }^{a}\end{array}$} & \multicolumn{2}{|c|}{$\begin{array}{c}\text { Prevalence of } \\
\text { T. gondii infection }\end{array}$} & \multirow{2}{*}{$\begin{array}{c}P \\
\text { value }\end{array}$} \\
\hline & & No. & $\%$ & \\
\hline \multicolumn{5}{|l|}{ Cats at home } \\
\hline Yes & 87 & 22 & 25.3 & 0.29 \\
\hline No & 86 & 28 & 32.6 & \\
\hline \multicolumn{5}{|c|}{ Cleaning cat excrement } \\
\hline Yes & 68 & 19 & 27.9 & 0.81 \\
\hline No & 98 & 29 & 29.6 & \\
\hline \multicolumn{5}{|l|}{ Traveled abroad } \\
\hline Yes & 17 & 1 & 5.9 & 0.01 \\
\hline No & 156 & 49 & 31.4 & \\
\hline \multicolumn{5}{|l|}{ National trips } \\
\hline Yes & 98 & 20 & 20.4 & 0.003 \\
\hline No & 73 & 30 & 41.1 & \\
\hline \multicolumn{5}{|c|}{ Pork consumption } \\
\hline Yes & 158 & 49 & 31 & 0.03 \\
\hline No & 15 & 1 & 6.7 & \\
\hline \multicolumn{5}{|c|}{ Goat meat consumption } \\
\hline Yes & 87 & 29 & 33.3 & 0.19 \\
\hline No & 86 & 21 & 24.4 & \\
\hline \multicolumn{5}{|c|}{ Mutton consumption } \\
\hline Yes & 49 & 11 & 22.4 & 0.23 \\
\hline No & 124 & 39 & 31.5 & \\
\hline
\end{tabular}

\begin{tabular}{ccccc}
\hline Turkey meat consumption & & & & \\
\hline Yes & 70 & 16 & 22.9 & 0.14 \\
\hline No & 103 & 34 & 33 &
\end{tabular}

Duck meat consumption

\begin{tabular}{ccccc}
\hline Yes & 24 & 9 & 37.6 & 0.31 \\
\hline No & 149 & 41 & 27.5 & \\
\hline Venison consumption & & & & \\
\hline Yes & 132 & 40 & 30.3 & 0.46 \\
\hline No & 41 & 10 & 24.4 & \\
\hline
\end{tabular}

Opossum meat consumption

\begin{tabular}{ccccc}
\hline Yes & 6 & 0 & 0 & 0.12 \\
\hline No & 167 & 50 & 29.9 & \\
\hline
\end{tabular}

Frequency of meat consumption

\begin{tabular}{lcccc}
\hline Never & 2 & 0 & 0 & 0.66 \\
\hline Up to 3 times a week & 161 & 47 & 29.2 & \\
\hline $4-7$ times a week & 10 & 3 & 30 &
\end{tabular}

Degree of meat cooking

\begin{tabular}{lcccc}
\hline Raw & 1 & 0 & 0 & 0.46 \\
\hline Undercooked & 8 & 1 & 12.5 & \\
\hline Well done & 164 & 49 & 29.9 & \\
\hline
\end{tabular}

Table 3 Bivariate analysis of selected putative risk factors for infection with $T$. gondii in migrant agricultural workers in Durango, Mexico (Continued)

\begin{tabular}{|c|c|c|c|c|}
\hline \multicolumn{5}{|l|}{ Raw milk consumption } \\
\hline Yes & 86 & 19 & 22.1 & 0.05 \\
\hline No & 87 & 31 & 35.6 & \\
\hline \multicolumn{5}{|l|}{ Sausages consumption } \\
\hline Yes & 145 & 44 & 30.3 & 0.34 \\
\hline No & 28 & 6 & 21.4 & \\
\hline \multicolumn{5}{|l|}{ Salami consumption } \\
\hline Yes & 37 & 9 & 24.3 & 0.48 \\
\hline No & 136 & 41 & 30.1 & \\
\hline \multicolumn{5}{|l|}{ Unwashed raw vegetables } \\
\hline Yes & 61 & 22 & 36.1 & 0.13 \\
\hline No & 111 & 28 & 25.2 & \\
\hline \multicolumn{5}{|l|}{ Unwashed raw fruits } \\
\hline Yes & 73 & 22 & 30.1 & 0.71 \\
\hline No & 98 & 27 & 27.6 & \\
\hline \multicolumn{5}{|l|}{ Untreated water } \\
\hline Yes & 108 & 34 & 31.5 & 0.34 \\
\hline No & 61 & 15 & 24.6 & \\
\hline \multicolumn{5}{|c|}{ Frequency of eating out of home } \\
\hline Up to 10 times a year & 123 & 43 & 35 & 0.008 \\
\hline$>10$ times a year & 48 & 7 & 14.6 & \\
\hline \multicolumn{5}{|l|}{ Soil contact } \\
\hline Yes & 165 & 49 & 29.7 & 0.27 \\
\hline No & 8 & 1 & 12.5 & \\
\hline \multicolumn{5}{|l|}{ Floor at home } \\
\hline Ceramic or Wood & 28 & 7 & 25 & 0.45 \\
\hline Concrete & 98 & 32 & 32.7 & \\
\hline Soil & 47 & 11 & 23.4 & \\
\hline
\end{tabular}

${ }^{\text {a }}$ Sums may not add up to 173 because of some missing values.

(20/122: 16.4\%). Other clinical characteristics were not associated with $T$. gondii IgM seropositivity.

Concerning behavioral characteristics (Table 3), the bivariate analysis showed a number of variables with a $P$ value equal to or less than 0.20 including traveling abroad $(P=0.01)$, national trips $(P=0.003)$, consumption of pork $(P=0.03)$, goat meat $(P=0.19)$, turkey meat $(P=0.14)$, opossum meat $(P=0.12)$, raw milk $(P=0.05)$, unwashed raw vegetables $(P=0.13)$, and a low frequency (up to 10 times a year) of eating out of home $(P=0.008)$. Other behavioral variables including animal contacts, consumption of raw or uncooked meat, and untreated water showed $P$ values higher than 0.20 in the bivariate analysis. Further analysis by using logistic regression (Table 4) showed that $T$. gondii exposure was positively associated with consumption of unwashed raw vegetables $(\mathrm{OR}=2.39 ; 95 \% \mathrm{CI}$ : 1.06-5.35; $P=0.03$ ) and low frequency of eating out of home $(\mathrm{OR}=3.87 ; 95 \% \mathrm{CI}: 1.43-10.42 ; P=0.007)$, and was 
Table 4 Multivariate analysis of selected characteristics of migrant agricultural workers and their association with T. gondii infection

\begin{tabular}{lccc}
\hline Characteristic & $\begin{array}{c}\text { Odds } \\
\text { ratio }\end{array}$ & $\begin{array}{c}\mathbf{9 5 \%} \\
\text { confidence interval }\end{array}$ & $\begin{array}{c}\boldsymbol{P} \\
\text { value }\end{array}$ \\
\hline Travel abroad & 0.17 & $0.02-1.51$ & 0.11 \\
\hline National trips & 0.30 & $0.13-0.65$ & 0.003 \\
\hline Consumption of: & & & \\
\hline$\quad$ Pork & 6.25 & $0.65-60.01$ & 0.11 \\
\hline$\quad$ Goat meat & 1.83 & $0.82-4.05$ & 0.13 \\
\hline$\quad$ Turkey meat & 0.47 & $0.21-1.06$ & 0.07 \\
\hline$\quad$ Raw milk & 0.40 & $0.18-0.87$ & 0.02 \\
\hline$\quad$ Unwashed raw vegetables & 2.39 & $1.06-5.35$ & 0.03 \\
\hline $\begin{array}{l}\text { Low frequency of eating out } \\
\text { of home }\end{array}$ & 3.87 & $1.43-10.42$ & 0.007 \\
\hline
\end{tabular}

negatively associated with national trips $(\mathrm{OR}=0.30$; 95\% CI: $0.13-0.65 ; P=0.003$ ) and consumption of raw milk $(\mathrm{OR}=0.40 ; 95 \% \mathrm{CI}: 0.18-0.87 ; P=0.02)$. Consumption of opossum meat was not included in the multivariate analysis because none of the 6 consumers were positive for anti- $T$. gondii antibodies.

\section{Discussion}

The 28.9\% seroprevalence of $T$. gondii exposure found in migrant agricultural workers is comparable with those (22.4\%-30.3\%) found in other rural communities in Durango, Mexico, including the general population in 3 rural communities [8], Mennonites [9] and Tepehuanos [13]. However, the seroprevalence found in migrant workers is higher than seroprevalences reported in urban populations in Durango City. The seroprevalence of T. gondii infection ranged between $6.1 \%$ and $12 \%$ among pregnant women [14], healthy blood donors [15], subjects suffering from a number of underlying diseases [16], and the general population in Durango City [10]. It is likely that differences in sanitation between urban and rural communities may contribute to the differences in seroprevalences. All migrant agricultural workers live in poverty and have poor housing conditions including soil floors at home and lack of urban services including potable water and drainage. In addition, when they work in seasonal agricultural labor out of their homes they live in shelters with very low hygiene and sanitary conditions too. To our knowledge there are no previous study on internal migrant agricultural workers in other Mexican states or other countries, therefore, we cannot compare our results with those of other studies. In a regional context, the seroprevalence of $T$. gondii infection found in migrant agricultural workers in Durango is higher than those (20.3\%-23.5\%) found in neighboring Mexican states (Chihuahua, Coahuila and Zacatecas) in a national seroepidemiology survey [17]. However, comparison of these seroprevalences should be interpreted with care since different laboratory methods among the studies were used. We used an enzyme immunoassay while an indirect immunofluorescence test was used in the national survey. In a national context, the seroprevalence of $T$. gondii infection in migrant agricultural workers in Durango is comparable with the mean national seroprevalence $(27.97 \%)$ of T. gondii infection reported in Mexico [18].

Of the behavioral characteristics, $T$. gondii exposure was positively associated with consumption of unwashed raw vegetables. This finding indicates that parasite transmission might have occurred by ingesting $T$. gondii oocysts. Shelters for migrant workers had cats. However, it is not clear whether infection occurred during their stay in the shelters or elsewhere. Transmission by parasite cysts was less likely to occur; migrant workers do not eat meat frequently because they cannot afford to buy it. The very high seroprevalence of anti-T. gondii IgM antibodies found in migrant workers suggests recent infections. Nevertheless, interpretation of the increased IgM seroprevalence should be taken with care since tests for detection of IgM antibodies may have a high rate of false positive results caused by limitations in test specificity or persistence of IgM antibodies [19]. Some known clinical features of toxoplasmosis including visual impairment and headaches [2,20-22] were observed in some migrant workers. In fact, frequent headaches showed a borderline $(P=0.05)$ association with $T$. gondii seropositivity. Of note, seropositivity to both anti-T. gondii IgM and IgG antibodies were associated with memory impairment. This association has been previously reported in gardeners [23]. In addition, we found an association of seropositivity to anti- $T$. gondii IgG antibodies with dizziness or syncope. Altogether, results indicate that both recent and latent toxoplasmosis cases were present among migrant agricultural workers. The impact of $T$. gondii on the health of migrant workers may have important labor implications. Migrant workers are paid for piecework; therefore, workers suffering from symptomatic toxoplasmosis may be affected by a reduction in their performance and income. Furthermore, T. gondii infection in workers is of concern since such infection has been associated with reflex impairment [24] and work accidents [25].

The positive associations of a low frequency of eating out of home and consumption of unwashed raw vegetables, and the negative association with national trips suggest that infection was most likely acquired at home in Durango by eating contaminated vegetables. Therefore, health care providers should focus their attention on educational strategies aimed to improve food hygiene practices in migrant agricultural workers. The negative association of T. gondii seropositivity with consumption of raw milk suggests that this behavioral characteristic did not play an important role in the transmission of $T$. gondii infection in migrant agricultural workers. 
A limitation of the study was that only one Mexican state was explored. However, results of this first study represent the baseline information on the seroprevalence and contributing factors for $T$. gondii infection in internal migrant agricultural workers in Mexico and other countries. Results of the present work are of concern since migrant agricultural workers play an important role in the economy of many countries. Results warrant for further research.

\section{Conclusions}

We conclude: 1) the seroprevalence of $T$. gondii exposure in migrant agricultural workers living in poverty is comparable to that reported in other rural populations in the region; however, seroprevalence is higher than that reported in the nearby urban capital city; 2) Toxoplasma may impact on the health of migrant workers. Our results may have labor implications and will help to design optimal preventive measures against $T$. gondii infection.

\section{Competing interests}

The authors declare that they have no competing interests.

\section{Authors' contributions}

CAE conceived and designed the study protocol, participated in the coordination and management of the study, applied the questionnaires, performed the laboratory tests and data analysis, and wrote the manuscript. FCR obtained clinical data, applied the questionnaires and performed the data analysis. OL performed the data analysis, and wrote the manuscript. All authors read and approved the final version of the manuscript.

\section{Author details}

'Laboratorio de Investigación Biomédica, Facultad de Medicina y Nutrición, Universidad Juárez del Estado de Durango. Avenida Universidad S/N, Durango, Dgo 34000, México. ${ }^{2}$ Servicios de Salud de Durango. Independencia y Puebla S/N, Canatlán, Durango 34450, México. ${ }^{3}$ Institute for Microbiology and Hygiene, Campus Benjamin Franklin, Charité Medical School, Hindenburgdamm 27, Berlin D-12203, Germany. ${ }^{4}$ Present address: Roche Molecular Diagnostics, Pleasanton, CA, USA.

Received: 5 March 2013 Accepted: 17 April 2013

Published: 20 April 2013

\section{References}

1. Dubey JP: Toxoplasmosis of animals and humans. Boca Raton, Florida: Second Edition. CRC Press; 2010.

2. Montoya JG, Liesenfeld O: Toxoplasmosis. Lancet 2004, 363:1965-1976.

3. Walker M, Zunt JR: Parasitic central nervous system infections in immunocompromised hosts. Clin Infect Dis 2005, 40:1005-1015.

4. Balasundaram MB, Andavar R, Palaniswamy M, Venkatapathy N: Outbreak of acquired ocular toxoplasmosis involving 248 patients. Arch Ophthalmol 2010, 128:28-32.

5. Jackson MH, Hutchison WM, Siim JC: A seroepidemiological survey of toxoplasmosis in Scotland and England. Ann Trop Med Parasitol 1987, 81:359-365

6. Bartolomé Alvarez J, Martínez Serrano M, Moreno Parrado L, Lorente Ortuño S, Crespo Sánchez MD: Prevalence and incidence in Albacete, Spain, of Toxoplasma gondii infection in women of childbearing age: differences between immigrant and non-immigrant (2001-2007). Rev Esp Salud Publica 2008, 82:333-342.

7. Chan BT, Amal RN, Hayati Ml, et al: Seroprevalence of toxoplasmosis among migrant workers from different Asian countries working in Malaysia. Southeast Asian J Trop Med Public Health 2008, 39:9-13.

8. Alvarado-Esquivel C, Cruz-Magallanes HM, Esquivel-Cruz R, Estrada-Martínez S, Rivas-González M, Liesenfeld O, Martínez-García SA, Ramírez E, TorresCastorena A, Castañeda A, Dubey JP: Seroepidemiology of Toxoplasma gondii infection in human adults from three rural communities in Durango State, Mexico. J Parasitol 2008, 94:811-816.

9. Alvarado-Esquivel C, Rojas-Rivera A, Estrada-Martínez S, Sifuentes-Álvarez A, Liesenfeld O, García-López CR, Dubey JP: Seroepidemiology of Toxoplasma gondii infection in a Mennonite community in Durango State, Mexico. J Parasitol 2010, 96:941-945.

10. Alvarado-Esquivel C, Estrada-Martínez S, Pizarro-Villalobos H, Arce-Quiñones M, Liesenfeld O, Dubey JP: Seroepidemiology of Toxoplasma gondii infection in general population in a northern Mexican City. $J$ Parasitol 2011, 97:40-43.

11. Alvarado-Esquivel C, Estrada-Martínez S: Toxoplasma gondii infection and abdominal hernia: evidence of a new association. Parasit Vectors 2011, 4:112.

12. Alvarado-Esquivel C, Torres-Berumen JL, Estrada-Martínez S, Liesenfeld $\mathrm{O}$, Mercado-Suarez MF: Toxoplasma gondii infection and liver disease: a casecontrol study in a northern Mexican population. Parasit Vectors 2011, 4:75.

13. Alvarado-Esquivel C, Estrada-Martínez S, García-López CR, Rojas-Rivera A, Sifuentes-Álvarez A, Liesenfeld O: Seroepidemiology of Toxoplasma gondii infection in Tepehuanos in Durango, Mexico. Vector Borne Zoonotic Dis 2012, 12:138-142.

14. Alvarado-Esquivel C, Sifuentes-Álvarez A, Narro-Duarte SG, Estrada-Martínez S, Díaz-García JH, Liesenfeld O, Martínez-García SA, Canales-Molina A: Seroepidemiology of Toxoplasma gondii infection in pregnant women in a public hospital in northern Mexico. BMC Infect Dis 2006, 6:113.

15. Alvarado-Esquivel C, Mercado-Suárez MF, Rodriguez-Briones A, Fallad-Torres L, Ayala-Ayala JO, Nevarez-Piedra L, Duran-Morales E, Estrada-Martínez S, Liesenfeld O, Márquez-Conde JA, Martínez-García SA: Seroepidemiology of infection with Toxoplasma gondii in healthy blood donors of Durango. Mexico. BMC Infect Dis 2007, 7:75.

16. Alvarado-Esquivel C, Liesenfeld O, Torres-Castorena A, Estrada-Martínez S, Urbina-Álvarez JD, Ramos-de la Rocha M, Márquez-Conde JA, Dubey JP: Seroepidemiology of Toxoplasma gondii infection in patients with vision and hearing impairments, cancer, HIV, or undergoing hemodialysis in Durango, Mexico. J Parasitol 2010, 96:505-508.

17. Velasco-Castrejón O, Salvatierra-Izaba B, Valdespino JL, Sedano-Lara AM, Galindo-Virgen S, Magos C, Llausás A, Tapia-Conyer R, Gutiérrez G, Sepúlveda J: Seroepidemiology of toxoplasmosis in Mexico. Salud Publica Mex 1992, 34:222-229.

18. Galvan-Ramirez Mde L, Troyo R, Roman S, Calvillo-Sanchez C, BernalRedondo R: A systematic review and meta-analysis of Toxoplasma gondii infection among the Mexican population. Parasit Vectors 2012, 5:271.

19. Liesenfeld O, Press C, Montoya JG, et al: False-positive results in immunoglobulin M (lgM) Toxoplasma antibody tests and importance of confirmatory testing: the Platelia Toxo IgM test. J Clin Microbiol 1997, 35:174-178

20. Uneke CJ, Duhlinska DD, Njoku MO, Ngwu BA: Seroprevalence of acquired toxoplasmosis in HIV-infected and apparently healthy individuals in Jos, Nigeria. Parassitologia 2005, 47:233-236.

21. Prandota J: Recurrent headache as the main symptom of acquired cerebral toxoplasmosis in nonhuman immunodeficiency virus-infected subjects with no lymphadenopathy: the parasite may be responsible for the neurogenic inflammation postulated as a cause of different types of headaches. Am J Ther 2007, 14:63-105.

22. Silva CS, Neves Ede S, Benchimol El, Moraes DR: Postnatal acquired toxoplasmosis patients in an infectious diseases reference center. Braz $J$ Infect Dis 2008, 12:438-441.

23. Alvarado-Esquivel C, Liesenfeld O, Márquez-Conde JA, Estrada-Martínez S, Dubey JP: Seroepidemiology of infection with Toxoplasma gondii in workers occupationally exposed to water, sewage, and soil in Durango, Mexico. J Parasitol 2010, 96:847-850.

24. Alvarado-Esquivel C, Estrada-Martínez S, Liesenfeld O: Toxoplasma gondii infection in workers occupationally exposed to unwashed raw fruits and vegetables: a case control seroprevalence study. Parasit Vectors 2011, 4:235.

25. Alvarado-Esquivel C, Torres-Castorena A, Liesenfeld O, Estrada-Martínez S, Urbina-Álvarez JD: High seroprevalence of Toxoplasma gondii infection in a subset of Mexican patients with work accidents and low socioeconomic status. Parasit Vectors 2012, 5:13.

doi:10.1186/1756-3305-6-113

Cite this article as: Alvarado-Esquivel et al: Seroepidemiology of infection with Toxoplasma gondii in migrant agricultural workers living in poverty in Durango, Mexico. Parasites \& Vectors 2013 6:113. 